\title{
Intermédialités
}

Histoire et théorie des arts, des lettres et des techniques

Intermediality

History and Theory of the Arts, Literature and Technologies

\section{Les rythmes contagieux d'une danse noire : le cake-walk}

\section{Rae Beth Gordon}

Numéro 16, automne 2010

rythmer

rhythmize

URI : https://id.erudit.org/iderudit/1001956ar

DOI : https://doi.org/10.7202/1001956ar

Aller au sommaire du numéro

Éditeur(s)

Revue intermédialités (Presses de l’Université de Montréal)

ISSN

1705-8546 (imprimé)

1920-3136 (numérique)

Découvrir la revue

Citer cet article

Gordon, R. B. (2010). Les rythmes contagieux d'une danse noire : le cake-walk Intermédialités / Intermediality, (16), 57-81. https://doi.org/10.7202/1001956ar
Résumé de l'article

Cette étude propose une anthropologie du geste d'une danse noire américaine, le cake-walk. En retraçant la façon dont le cake-walk a été transformé par des artistes français, cette analyse souligne la spécificité française des perceptions de cette danse qui est vite devenue un phénomène de société. On remarque la convergence entre deux champs scientifiques, vulgarisés et largement répandus dans la presse : la psycho-pathologie et la théorie de l'évolution. Le discours autour du mouvement hystérico-épileptique et celui autour de la régression darwinienne sont superposés dans presque tous les commentaires sur les rythmes entraînants et les gestes du cake-walk, créant des inquiétudes sur la contagion et la dégénérescence. 


\title{
Les rythmes contagieux d'une danse noire: le cake-walk
}

\author{
Rae Beth Gordon
}

L'art de la danse est aujourd'hui bien différent de ce qu'il fut. Depuis quelques années surtout, il a fait de très grands progrès. De complication en complication, la chorégraphie est allée chercher ses modèles, ou du moins ses inspirations, jusque dans les sarabandes des peuplades sauvages. C'est ainsi que [...] deux remarquables artistes américains, les Elks, ont importé le célèbre cake-walk.

On peut dire qu'une société se traduit au naturel par sa danse favorite. [...] Le tam-tam n'est pas un miroir moins fidèle du peuple noir que le menuet [ne l'est du $18^{e}$ siècle français] $]^{1}$.

a performance ainsi que la réception du spectacle noir portent en France la - marque de l'ambivalence des sentiments du public envers les Africains et les Noirs américains. Les villages africains reconstitués, entre 1877 et 1912, au Jardin d'Acclimatation, de même que les spectacles de danse africaine présentés lors des Expositions universelles de 1889 et 1900 ont attiré des milliers de Français, et ont suscité des réactions de surprise devant l'étrangeté provoquée par les corps noirs en mouvement. Alors que la popularité des danseurs africains, des danseurs de cake-walk, des black-face minstrels ${ }^{2}$ au music-hall et au café-concert, ainsi que celle de Chocolat au Nouveau-Cirque, se basaient avant tout sur des stéréotypes raciaux négatifs, d'autres attributs de ce que les anglophones nomment blackness, cette fois connotés positivement, ont émergé. Il s'agissait des éléments de rythme et de gestuelle qui contenaient et communiquaient une vitalité et une essence

1. Extraits de Paris qui chante, 31 janvier 1904 et de Paul Bourgette (pseudonyme de Louis Sonolet), Fantasio, 15 mars 1910.

2. Les premiers minstrel shows en France datent de la fin des années 1870 ; ils sont réalisés par des Noirs dont le maquillage accentue les yeux et crée une bouche énorme, ou bien par des Blancs en black-face, c'est-à-dire grimés en noir. 
que les Français avaient envie de s'approprier. L'apparition du cake-walk en 1902 témoigne de cette réception ambivalente.

À partir de 1878 environ, le darwinisme se vulgarise et se répand dans la presse bourgeoise et populaire; cette vision d'un retour à un état primitif de l'humanité vient ajouter un nouveau frisson aux fantasmes inspirés par l'Autre. Dans un ouvrage antérieur, j’ai souligné la convergence temporelle, en France, entre les performances de la danse africaine et la médiatisation des symptômes d'hystéro-épilepsie et ce pas seulement parce que ces groupes étaient considérés comme dégénérés par la médecine et par les anthropologues, mais en raison des parallèles explicites que l'on retrouve dans le répertoire de mouvements des deux groupes ${ }^{3}$. Cette similarité n’a pas échappé aux intellectuels ni au grand public: lors de l'Exposition universelle de 1889, les danseurs algériens en transe, les Aïssaouas, font dire à Eugène Melchior de Vogüié qu'ils «accomplissent leurs mômeries sans se douter que nous avons bien mieux à la Salpêtrière ${ }^{4}$ ». De fait apparaît un nouveau personnage au café-concert en 1875: celui de la chanteuse épileptique. Le style se répand au moment même où les cas d'hystéroépilepsie se multiplient de façon spectaculaire, le genre épileptique exploitant la médiatisation de ce phénomène de société qu'est l'hystérie' (Fig. 1). En effet, les numéros de «caf'conc' » sont très influencés par le discours médical ambiant sur les pathologies nerveuses et par les descriptions qu'on peut lire dans les journaux et revues de l'époque. En 1900, Mistinguett chante «La Parisienne épileptique» qui commence ainsi : "Quand j’entends la musique, je deviens épileptique. » La chanteuse épileptique la plus célèbre était la grande amie de Colette, Polaire, qui chantait avec « des gestes cassés... qui s'arrêtaient aux limites de l'épilepsie ${ }^{6}$ ». En effet, "pour une bonne moitié, la chanson à succès de ce temps-ci relève du trémoussoir de feu Charcot. Elle trépide. Elle a l'hystérie gesticulatoire.7 »

3. Voir Rae Beth Gordon, Dances with Darwin, 1875-1910: Vernacular Modernity in France, Burlington et Farnham, Ashgate Press, 2009. Le présent article est en partie issu des chapitres 4 et 5 de ce livre.

4. Eugène Melchior de Vogüé, «À travers l'exposition », Revue des Deux-Mondes, 15 septembre 1889 , p. 454.

5. L'hystérie est confondue avec l'épilepsie dans l'imaginaire populaire, et le terme d'hystéro-épilepsie est utilisé dans les milieux médicaux jusqu'au $20^{\mathrm{e}}$ siècle.

6. Robert Dieudonné, «Du café-concert à la Renaissance», Femina, septembre 1906, dans BnF Dossier Ico Per 21238 (1).

7. Georges Montorgueil et al., Les demi-cabots: le café-concert, le cirque, les forains, Paris, G. Charpentier et E. Fasquelle, 1896. Les rapports entre l'hystéro-épilepsie et la gestuelle au café-concert, puis dans le cinéma comique en France sont analysés en détail 
Ainsi, à partir de 1875, les mouvements frénétiques, saccadés et mécaniques, accompagnés de secousses, de contorsions bizarres, de dislocations, de tics et de grimaces remportent un succès considérable au café-concert. Cette chorégraphie ressemble à s'y méprendre à une pathologie nerveuse rattachée par Charcot en 1878 à l'hystéro-épilepsie, la chorée. Cette observation publiée dans L'iconographie photographique de la Salpêtrière aurait tout à fait pu se rapporter à la performance de l'une de ces chanteuses: «Elle trépide, saute, danse convulsivement, gesticule, grimace, et tire la langue.» Dans la chorée rythmique, proche du délire saltatoire, «les malades sautent de manière rythmique, absolument comme si

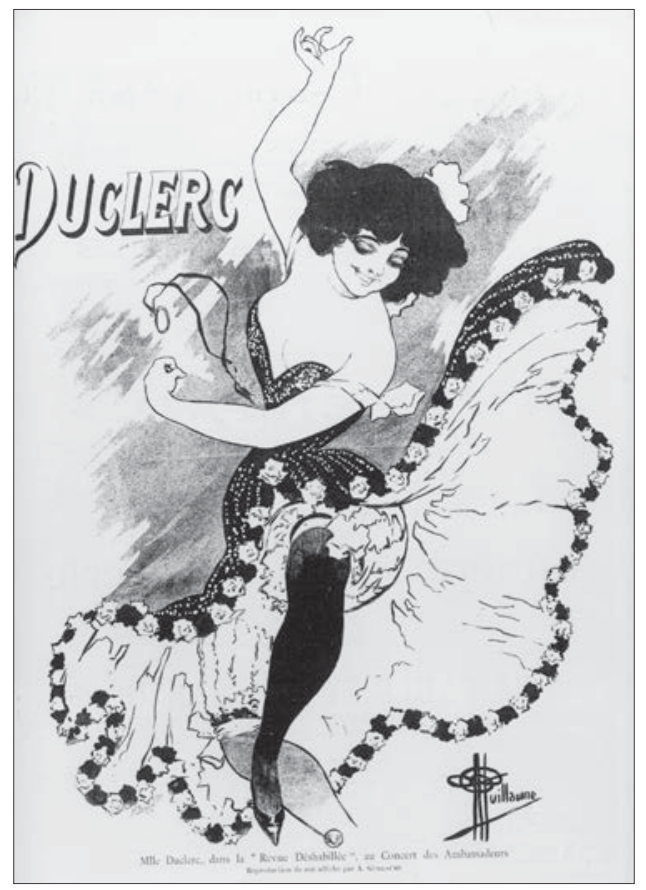

Fig. 1: Duclerc par Albert Guillaume, avec la gracieuse permission de la Bibliothèque Forney, Paris.

dans Rae Beth Gordon, Why the French Love Jerry Lewis: From Cabaret to Early Cinema, Stanford, Stanford University Press, 2001, et plus récemment dans «Les galipettes de l'autre burlesque», dans Laurent Guido et Laurent Le Forestier (dir.), «Aux sources du burlesque cinématographique: les comiques français des premiers temps », 1895, n 61, 2010, p. 129-148. On retrouve dans Gordon, 2009, les analyses d'une gestuelle perçue comme hystérique ou épileptique dans les spectacles de danse noire en France. 
elles dansaient ${ }^{8} »$. Comme nous aurons l'occasion de le voir, les mouvements du cake-walk seront rapidement dénigrés en raison de leur proximité avec les gesticulations épileptiques. C'est la raison pour laquelle il est particulièrement intéressant d'étudier les spécificités de la réception de cette danse en France. Non seulement y trouve-t-on les entrecroisements entre l'image du Sauvage et les représentations de l'Hystérique, mais à cette interférence intermédiale entre médecine, ethnologie et danse, il faut ajouter une complication supplémentaire: les chanteuses épileptiques, en s'emparant du cake-walk, en ont exagéré les poses et les mouvements et, ce faisant, l'ont transformé en performance épileptique.

Les mouvements excessifs ou étranges et les rythmes inattendus, qu'ils proviennent de danseurs africains, de minstrels et danseurs noirs américains, de chanteuses épileptiques, ou (par le biais de la presse illustrée) des hystériques, excitent le public. Ces exhibitions spectaculaires - toutes simultanément très médiatisées - du corps en mouvement atteignant un public de masse, des amalgames ne peuvent manquer de se créer dans l'imagination populaire. Les descriptions journalistiques en témoignent: c'est précisément ce qui se produit, puisque ces spectacles ont, entre autres, pour effet d'inspirer la peur d'une contagion à travers le geste.

Les fantasmes reliés à la sexualité des Africains sont monnaie courante à la Belle Époque, appuyés par la profusion de photographies d'Africaines aux seins nus dans les magazines populaires. La sexualité, la brutalité, et même le cannibalisme, considéré comme le plus primitif de leurs traits sauvages, accompagnent alors presque chaque portrait des Africains, et alimentent les fantasmes sexuels qui leur sont liés: "Au son d'un orchestre barbare [...] les danseuses esquissent des pas lascifs et provoquants» pour accompagner un défilé de jeunes vierges devant leurs prétendants. «Certaines Noires çà et là sont anthropophages. Mais leur charme n'en est que plus vif, à ce qu'on dit ${ }^{9}$.» Barbarie et Africains dansant frénétiquement semblent aller de pair: «Les spectacles donnés par les Achantis dénotent une véritable barbarie dans ce peuple africain. [Ils ont] les coutumes les plus sauvages $[. .$.$] des têtes coupées partout [...]. Le fétichisme$ le plus monstrueux et extravagant est la base de leur religion avec ses sacrifices

8. Désiré-Magloire Bourneville et Paul Régnard, Iconographie photographique de la Salpêtrière, vol. 2, Paris, Bureaux du «Progrès médical», 1878, p. 35-36; Dimitri Ivan Michailowski, Nouvelle iconographie de la Salpêtrière, vol. 5, Paris, Bataille et Co., 1892, p. 65-66. Le dernier tome de la Nouvelle iconographie date de 1918.

9. Henri de Weindel, «Le charme noir», Fantasio, $1^{\text {er }}$ septembre 1906, p. 100 (je souligne). 
humains ${ }^{10}$.» Notons surtout l'impact sur le corps du spectateur dans cette description de 1887 : «ces danses sont pénibles à voir, les mouvements en sont trop rapides et trop violents. La bête y cherche sa joie dans la détente éperdue de ses muscles [sans se soucier] de l'harmonie des évolutions et de l'équilibre des lignes déplacées. On n'y sent que le déploiement aveugle des forces animales ${ }^{11}$.»

Les expériences psychophysiques et psychophysiologiques de la fin du $19^{\mathrm{e}}$ siècle - notamment celles de Charles Henry qui publie son Introduction à une esthétique scientifique en 1885 - ont démontré que la vue de mouvements pouvait engendrer une répétition inconsciente dans le corps du spectateur, c'està-dire dans son système vasculaire, musculaire, et respiratoire. La psychophysiologie de l'imitation inconsciente trouve son complément dans les expériences psychologiques sur la suggestion et l'imitation inconsciente de l'École de Nancy. Le sociologue Gabriel Tarde écrit en 1890 qu'il n'y a «rien de plus vulgarisé que [ces notions]. Une action nous donne [...] l'idée plus ou moins irréfléchie de l'imiter ${ }^{12}$. À la même époque, l'esthéticien Paul Souriau écrit que les mouvements convulsifs de la danse "peuvent par une sorte de contagion provoquer dans celui qui regarde des symptômes similaires ${ }^{13}$ ». L'impact des gestes des artistes sur le spectateur n’a pas échappé aux chroniqueurs de spectacle, qui en font très souvent la remarque. Qui plus est, les recherches menées ces vingt-cinq dernières années dans les neurosciences sur les «neurones miroirs », dont celles

10. «Les Achantis au Jardin d'Acclimatation », Paris illustré, 24 septembre 1887, p. 6. Même les reportages sur la danse africaine publiés dans la presse spécialisée et mondaine expriment ces peurs et fantasmes: dans les parties les plus reculées du continent africain, on trouve «les races les plus inférieures $[. .$.$] une race déchue ou incomplètement déve-$ loppée, on ne sait, a un talent particulier pour les rythmes inattendus et la bizarrerie, je dirais presque la sauvagerie [qui] leur prête un charme particulier». Julien Tiersot, «Notes d'ethnographie musicale: le continent africain », Le ménestrel, 22 mars 1903, p. 89.

11. Citation non attribuée dans Raymond Bachollet, Jean-Barthélemi Debost, AnneClaude Lelieur et al. (dir.), Négripub: l'image des noirs dans la publicité, Paris, Somogy, 1992, p. 44 .

12. Gabriel Tarde, Les lois de l'imitation [189o], Paris, Kimé, 1993, p. 87 et 84.

13. Paul Souriau, L'esthétique du mouvement, Paris, Alcan, 1889, p. 120. Le propos du $\mathrm{D}^{\mathrm{r}}$ Paul-Max Simon est plus hyperbolique: « on mettait son système nerveux en unisson avec les trépidations de la musique d'Offenbach et l'on devenait immanquablement hystérique». Dr Paul-Max Simon, L'hygiène de l'esprit pour la préservation des maladies mentales et nerveuses, Paris, Baillière, 1877, p. 98. La théorie darwinienne se réfère elle aussi à la nature biologique et instinctuelle de l'imitation. 
menées par Giacomo Rizzolato et son équipe à l'Université de Parme en 1996, ont confirmé le bien-fondé de ces travaux effectués un siècle auparavant ${ }^{14}$.

La danse africaine se transforme très vite en attraction de music-hall : à partir de 1878 , des mises en scène de rites africains sont jouées à côté des danses aux Folies Bergère, au Casino de Paris, au Théâtre de la Porte Saint-Martin, et au Châtelet. Ces spectacles sensationnalistes coïncident avec la première vague d'expansion coloniale, amorcée en 1879. Les plus spectaculaires sont La Vénus noire d'Adolphe Bélot au Châtelet en 1879, La conquête de Dahomey, également au Châtelet, qui rivalise avec Au Dahomey au Théâtre de la Porte Saint-Martin, et avec le grand spectacle dahoméen au Casino de Paris qui attire plus de 300 ooo spectateurs (les trois représentations datent de 1892). Enfin, il faut citer «L'Exposition d'ethnographie coloniale» au Champ de Mars avec 150 Dahoméens - chefs, guerriers et amazones de 1893. Dans cette même période, les dessins et textes satiriques stéréotypant les Africains font la joie des lecteurs de journaux et de magazines, tels que Le courrier français, Fantasio, L'illustration, Le monde illustré, et Le rire (au rythme d'un numéro sur trois!). Naturellement, les images construites à la fin du $19^{\mathrm{e}}$ siècle, «confortées » pour la plupart par l'anthropologie et l'ethnologie contemporaines, sont essentielles pour comprendre l'impact du cake-walk en 1902, et la menace qu'il était censé représenter pour la société française. Elles servent également de toile de fond à La revue nègre avec Joséphine Baker en 1925.

Certes, la vue de visages peints, de scarifications, de tatouages, et de corps nus recouverts d'un simple pagne étonne les spectateurs. Mais je voudrais insister sur le fait que ce qui les tient en haleine, les fascine et les émerveille, c'est la façon dont ces corps se meuvent. L'ethnologue John Szwed a d'ailleurs fait remarquer en 1975 que, dans les premiers contacts occidentaux avec l'Autre racial : « it was the use of their bodies that had the most impact - motor habits, the way one moves, laughs, etc. ${ }^{15}$ ". Il y a une étrangeté irréductible dans la façon dont le corps noir évolue à des rythmes qui ne sont pas familiers. Ce sont non seulement les mouvements de danse, mais également «l'extravagance de leur marche, une sorte de boiterie cadencée [et] les changements incroyables de physionomie » qui

14. Pour les expériences psycho-physiques-physiologiques en rapport avec la médecine et le spectacle populaire, voir Gordon, 2001, p. 8-12, et Gordon, 2009, p. 105-116. Pour une appréciation des recherches sur les neurones miroirs, voir Giacomo Rizzolatti et Corrado Sinigaglia, Les neurones miroirs, trad. Marilène Raiola, Paris, Odile Jacob, 2008.

15. John F. Szwed, «Race and the Embodiment of Culture», Ethnicity, vol. 2, n ${ }^{\circ}$, mars 1975 , p. 23. 
sont remarquables aux yeux des observateurs français ${ }^{16}$. L'écrivain Jules Lemaître perçoit ainsi les Achantis:

Tandis que ces sauvages dansaient, je me répétais malgré moi la vieille réflexion [...] qu'il y a plus de différence d'homme à homme que d'animal à homme. Allez voir ces fils monstrueux de l'Afrique équatoriale: vous aurez sûrement l'impression que l'abîme est moindre entre les bons chiens qui jappent près de là et un Achanti, qu'entre un Achanti et M. Taine ou M. Herbert Spencer ${ }^{17}$.

Le choix des deux théoriciens évolutionnistes comme éléments de comparaison est savoureux.

Le tam-tam est père des bonds formidables, des contorsions ahurissantes, des grimaces hilarantes, des entrechats frénétiques. Le tam-tam, c'est la synthèse de la vie nègre. [...] [Le danseur] se ramasse [en] nain, puis se détend, saute sur place, se contorsionne, se disloque, $[\ldots]$ avec des gestes et des grimaces de singe. Une femme lui succède [avec] la folle détente de ses jambes nerveuses [et] la souple légèreté d'une antilope. Dans ses gestes et ses attitudes, il y a quelque chose de brusque, de sauvage, d'animal même qui prouve que la légèreté n'est pas la grâce ${ }^{18}$.

L'instinct et le mouvement excessif apparaissent indissolublement liés, et ensemble, ils constituent le signe d'un stade inférieur d'évolution. «The lower we descend in the scale of animal life, the more prominent do the motor reactions become. This life is primarily of the instinctive type ", écrit le psychologue Boris Sidis $^{19}$. Cette observation vaut aussi bien pour les conceptions de l'hystérie et de l'épilepsie que pour celles qui s'appliquent à la danse africaine.

En dépit de ces perceptions, qui placent les Noirs au bas de l'échelle de l'évolution, la presse propose des poèmes et des dessins de minstrels noirs au caféconcert ou d'Africains en ville séduisant les femmes. Brunw (grimé comme un minstrel) chante dans «Viens à Timbuktu » (1907): "Viens, viens, à Timbuktu je t'embrasserai partout, mieux qu'un Blanc. » Quant à Anna Dancrey, elle entonne : «Je viens de San Francisco avec un beau Moricaud qui m’a troublée de bas en haut. C'qui m'affole, c'est son cake-walk. [...] Pour l'amour mon nègre est un vrai poème. » Dans une publicité pour le remontant Kola Marque, un jeune couple

16. E. P., «Chez l’Almamy Samory», Revue illustrée, 1886, p. 684.

17. Jules Lemaître, 19 septembre 1887, dans Jean-Pierre Biondi, Les anticolonialistes (1881-1962), Paris, Laffont, 1992, p. 32.

18. Paul Bourgette, «Le parfum de la femme noire: fêtes galantes », Fantasio, 15 mars 1910 , p. $563-564$.

19. Boris Sidis, «The Doctrine of Primary and Secondary Sensory Elements", Psychological Review, n 15, 1908, p. 51. 
parisien installé au café, l'homme hagard et neurasthénique, regarde un grand Soudanais passer fièrement. La femme demande: «Pourquoi ne prendriez-vous pas de la Kola Marque? Il paraît que c'est pour cela que les Soudanais et autres exotiques sont si solides et vigoureux. » En effet, Kola Marque est vendu comme remède contre la neurasthénie ${ }^{20}$. Ce n'est donc pas l'Africain qui est ici la victime de la dégénérescence mais le Français blanc, menacé par ce fléau, surtout depuis la défaite lors de la guerre franco-prussienne. L' Autre fascine. Pas seulement par son étrangeté mais par les fantasmes qui entourent les prouesses physiques et sexuelles du corps noir.

[Floradora est un spectacle] d'un tel exotisme qu'il ne peut manquer d'exciter et de retenir la curiosité parisienne, étrangement éprise de tout ce qui est étranger. [...] Un cake-walk formidable met la salle en délire $[\ldots]$ avec ses gestes simiesques ${ }^{21}$.

\section{LE CAKE-WALK}

Le tam-tam, on s'en souvient, est « un miroir fidèle du peuple noir » : c'est-à-dire que la danse est l'expression de l'ethos et de l'essence de la société qui l'a créée. Or, que se passe-t-il lorsqu'une société adopte les danses d'une autre?

Lorsqu'en novembre 1902, les Elks et leur troupe de danseurs noirs et blancs débutent au Nouveau-Cirque dans la revue-pantomime Les joyeux Nègres, les pas saccadés et le rythme syncopé font immédiatement du cake-walk la nouvelle folie du jour. Un engouement qui dure plus de trois ans malgré les efforts pour le détrôner et, surtout, pour le rendre méprisable - voire dangereux - en le stigmatisant comme une danse de sauvages marquée par des traits régressifs.

Le compte-rendu de la première laisse présager une «longue et superbe carrière pour Les Joyeux Nègres. [...] il n'y a «rien de plus original, de plus entraînant, de plus agréable à l'œil que ces nouvelles danses de cake-walk» et «la diversité et l'élégance des attitudes ont fait sensation ${ }^{22}$ ». À la tête de la troupe se trouvent M. et Mme Elks, des Blancs, suivis de deux couples noirs et deux enfants noirs, puis de deux femmes blanches. Il importe de souligner l'observation de l'élégance de la danse; elle est cruciale pour comprendre la façon dont l'audience se construit par la suite en France pour le cake-walk. Voici quelques commentaires anglo-saxons: «Miss Phillips' idea of the poetry

20. Le courrier français, 22 juillet 1900.

21. Raoul Ralph, «Bouffes-Parisiennes, Floradora», Le courrier français, ${ }^{\text {er }}$ février 1903, p. 4 (je souligne).

22. «Nouveau-Cirque - Les Joyeux Nègres», Le courrier français, 2 novembre 1902, p. 10 (je souligne). 
in motion in the cake-walk is wonderful to behold» (des U.S.A., 30 Avril 1898); «The Grand cake-walk, participated in by 16 devotees of Delsarte (who) stand ready to defend their claim as Champions on the merits of Style, Grace and

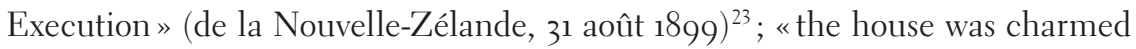
by this unique spectacle of Negro grace ${ }^{24}$ (de l'Australie, 9 septembre 1899). Grâce, élégance, poésie en mouvement... Comme nous allons le voir, la grâce allait vite disparaître en France, remplacée par la perception de mouvements épileptiques et d'attitudes contorsionnées, cédant à un glissement à rebours vers un stade antérieur de l'évolution.

Voici comment Jean Cocteau se souvient de cette première, trente-trois ans plus tard: «La salle trépignait, debout, et, au milieu de cette salle en délire, M. et Mme Elks dansaient. Ils dansaient, maigres, crochus [...] les genoux plus haut [sic] que le visage renversé en arrière [...] arrachant leurs gestes d'eux-mêmes [...]. Ils se cassaient en deux, en trois, en quatre ${ }^{25}$.» Dans la grande majorité des descriptions du cake-walk, on retrouve cette même insistance sur les rythmes entraînants qui communiquent leurs mouvements au public. Les spectateurs «avaient été à ce point mis en joie par [The Belle of New York], émoustillés [...] entraînés par l'extraordinaire mouvement [...] que d'aucuns sortaient en dansant involontairement la gigue, cependant que d'autres esquissaient de folâtres pas de cake-walk ${ }^{26}$ ».

À peine deux mois après la première au Nouveau-Cirque, l'hebdomadaire Paris qui chante s'exclame que «le cake-walk de couples nègres aux faces simiesques» est «le triomphe de la Bamboula américanisée avec toutes sortes de gestes dégingandés, de contorsions abracadabrantes, aux sons d'étranges polkas-marches, rapides, hérissés de contretemps et de syncopes ${ }^{27} »$. Le contraste avec la poetry in motion et avec la critique de la première au Nouveau-Cirque est frappant. Les raisons de cette divergence sont instructives. L'illustration du 10 janvier 1903 nous fournit un début d'explication :

23. François D. Delsarte (1811-1871) est un théoricien et professeur des études dramatiques et de mime qui a avancé la théorie d'un langage gestuel comme l'expression la plus directe de l'âme.

24. Articles de journaux reproduits dans Henry T. Sampson, The Ghost Walks: A Chronological History of Blacks in Show Business, 1865-1910, Metuchen, Scarecrow Press, 1998, p. 152, 185 et 194.

25. Jean Cocteau, Portraits-souvenir: 1900-1914, Paris, Grasset, 1935, p. 74. Cocteau se trompe sur la date de la première.

26. Raoul Ralph, «Moulin-Rouge : la belle de New York», Le courrier français, 7 juin 1903 , p. 4 .

27. «Le cake-walk des Négrillons », Paris qui chante, 31 janvier 1903. 
À vrai dire, les mimiques et les pas du cake-walk avaient bien été esquissés auparavant dans certains salons franco-américains, par quelques jeunes filles transatlantiques... du dernier bateau. Mais ce ne furent que de timides essais. Il manquait à cette chorégraphie nouvelle la consécration des cirques et des grands cafés-concerts. La lacune est [maintenant] comblée. Au Nouveau-Cirque d'abord, puis successivement dans tous les music-halls [...] se sont improvisées des troupes de nègres. [...] Et aussitôt $[\ldots]$ des professionnels [...] ont imaginé des variations acrobatiques sur le rythme originel. Les Parisiens s'imaginent connaître le cake-walk. Ils n'en ont pourtant - il faut bien le leur dire - qu'une caricature sur les planches des cafés-concerts. [...] En Amérique tout forme une harmonie de couleur locale ${ }^{28}$.

Les jeunes Américaines blanches qui avaient introduit le cake-walk dans les salons parisiens étaient «timides»; pour s'imprimer dans l'esprit des Parisiens et pour devenir la danse du moment, le cake-walk a besoin de l'indispensable "consécration des cirques et des grands café-concerts", et surtout de ces contorsions qui rappellent l'épilepsie. Par conséquent, il devient un phénomène de société grâce à des artistes comme Polaire, qui le rendent plus épileptique en créant des «variations acrobatiques» sur le rythme, mais également grâce à l'exotisme d'un spectacle noir. Or, Polaire, née en Algérie, est elle-même exotique, et ce fait est constamment souligné.

Cette chronologie nous permet d'esquisser un échange dynamique entre performance et société, entre Noir et Blanc, entre «haute» et «basse » culture. Les jeunes Américaines qui ont introduit la danse dans les salons parisiens n'ont eu, pour ainsi dire, aucun impact. Était-ce parce que leur prestation n’était qu'une pâle imitation? Parce qu'elles n'étaient pas noires? Ou bien parce qu'elles n'embellissaient la danse de poses hyperboliques? Chose certaine, dans cette rencontre initiale en France avec le cake-walk, il existe une insularité totale de classe (haute), de milieu (privé), et de race (blanche). Ensuite, on observe un changement de décor et de corps: la performance de la danse, dans ces lieux chargés d'excitations sensorielles que sont le cirque et le music-hall, produit au sein d'une foule de spectateurs socialement diversifiés un «délire » de réaction physiologique et d'imitation inconsciente. Leurs corps incorporent désormais le rythme et les poses du cake-walk: il s'opère un échange entre high et low, car le «Tout Paris » blanc est entraîné, secoué, excité par des performers noirs ou exotiques, comme l'Espagnole Caroline Otero, l'Espagnole juive Eugénie

28. C.C.B., «Le cake-walk», L'illustration, 10 janvier 1903, p. 30 (je souligne). Confirmation dans Le monde illustré: les théâtres exagèrent le cake-walk, «le faisant danser par Polaire, Mariette Sully et Balthy»; H. F., «La danse en vogue: le cake-walk», Le monde illustré, 21 février 1903, p. 185. 


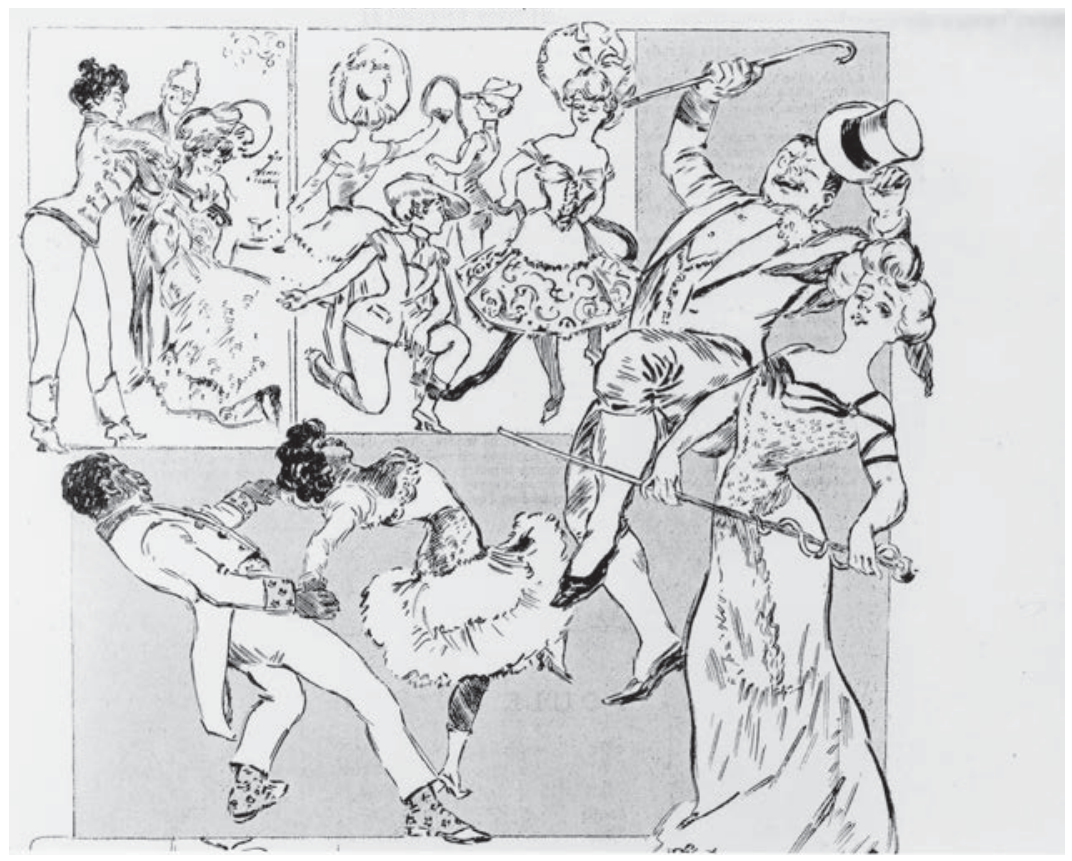

Fig.2: Le cake-walk à l'Olympia par Widhopff, avec la gracieuse permission de la Bibliothèque Forney, Paris.

Fougère, ou encore Polaire ${ }^{29}$. Cette dynamique est renforcée par les variations acrobatiques des artistes blanches, qui rendent la danse plus «épileptique », et qui sont parfois grimées en black-face comme cette danseuse à l'Olympia en 1903 (Fig. 2). C'est ainsi que les artistes blanches ont pu voler la vedette aux danseurs noirs. Les syncopes de la crise hystéro-épileptique se métamorphosent en secousses rythmiques irrégulières de musique syncopée $e^{30}$.

29. Willy (Henri Gauthier-Villars) écrit que Polaire était la première à le danser, déclarant que «le cake-walk prenait un rythme et une figure que les Américains ne reconnaissaient plus» (Willy, Danseuses, Paris, A. Méricant, 1904, p. 298). Des six films américains de cette danse visionnés à la Library of Congress, il n'y en a qu'un qui ressemble aux descriptions du mouvement hyperbolique en France: le Comedy Cake-Walk (1903); dans tous les autres, le mouvement est élégant et retenu.

30. La déviation d'une périodicité régulière a un effet certain sur le système nerveux. Andre Levinson ne dit pas autre chose quand il constate dans «The Negro Dance: Under European Eyes» que «the first aim of this kind of dance is an intense and penetrating reproduction of some rhythmic formula [...]. The primitive human instinct is violently affected by such rhythmic insistence» (p. $282-283$; je souligne). Andre Levinson, «The 
L'engouement pour l'Autre est explicite dans le compte-rendu de Floradora cité en exergue de cette section. Ce que les Parisiens ignorent, c'est que le cake-walk est né dans des plantations du sud des États-Unis et qu'il constitue à l'origine une parodie des menuets et gigues dansés par les Maîtres. Il est donc ironique que le cake-walk soit perçu comme une exhibition de traits régressifs, et, encore plus, que l'imitation d'une danse noire, imitant elle-même les manières de la haute société blanche, soit considérée comme dangereusement contagieuse.

Le cake-walk offre un exemple parfait de ce qu'on pourrait appeler un effet de ricochet culturel. La gestuelle noire, retravaillée et dénaturée par des artistes de music-hall blanches, altère la manière de voir des spectateurs. Elles font évoluer la danse vers une exhibition épileptique ${ }^{31}$, la transformant en même temps selon l'attente ethnologique des Français versés dans les classifications raciales d'Africains grâce à la presse, les expositions universelles et les zoos humains. Car la danse noire se doit d'être frénétique, violente, hyperbolique et excessive. Désormais, les jugements seront basés sur cette attente, comme on peut le voir dans Le monde illustré du 21 février: "Le cake-walk ne vaut que s'il est outré et si les gestes... perdent toute mesure ${ }^{32}$.» Le journaliste de L'illustration, cité plus haut, pouvait bien informer ses lecteurs que les Parisiens n'avaient «qu'une caricature [du cake-walk] sur les planches des café-concerts»!

C'est bien cette version hybride - celle des Noirs américains revisitée par les artistes de music-hall parisiens - que l'on aperçoit en 1903 dans Le cake-walk infernal de Georges Méliès. Méphisto retourne aux enfers après une visite sur terre, au music-hall dans lequel il a vu danser le cake-walk, et veut l'apprendre à ses diablotins. Arrive alors le couple de l'Olympia (Fig. 2) avec la frénésie époustouflante de ses mouvements disloqués et épileptiques. Mais Méphisto-Méliès ne s'en laisse pas démontrer : à épileptique, épileptique et demi! À l'aide de trucages,

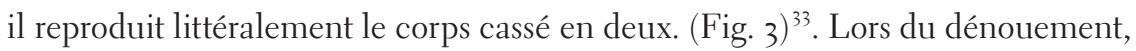
il ordonne aux diablotins de s'arrêter, mais ils en sont incapables. Dislocation,

Negro Dance: Under European Eyes», Theatre Arts Monthly, vol. 11, n 4, avril 1927, p. 282-293.

31. Désormais, il semble tout à fait naturel que ses meilleures interprètes appartiennent au genre de la chanteuse épileptique. Aucune danseuse noire n'est devenue célèbre en la dansant.

32. Le monde illustré du 21 février, p. 184.

33. Georges Méliès, Le cake-walk infernal, Star-Film, 453-457, Fechner Productions 2007; StudioCanal 2008. On peut voir ce film, soit dans le coffret double DVD produit par Christian Fechner et Studio Canal en 2008, soit sur YouTube (la mise en ligne du Cakewalk infernal date du 30 novembre 2010). 


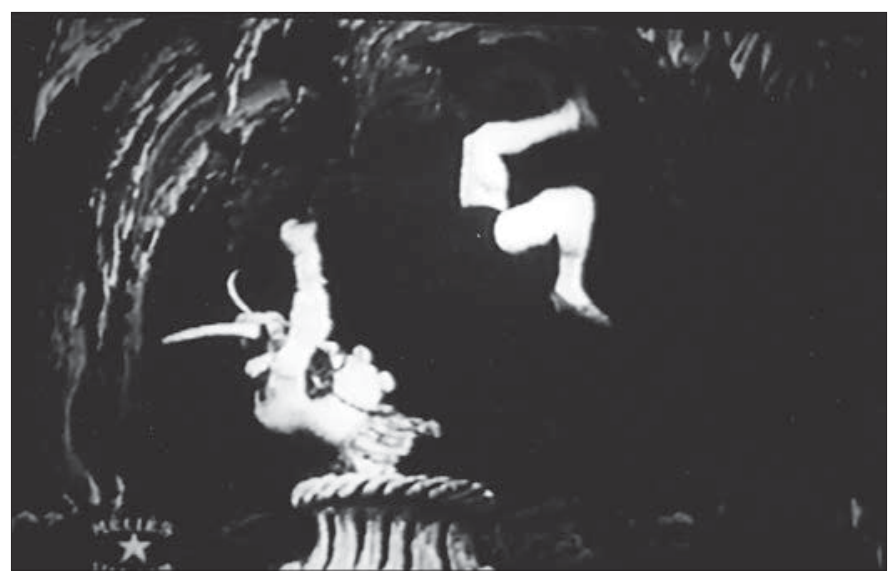

Fig.3: Georges Méliès, Le cake-walk infernal, 1903, collection de l'auteur.

épilepsie, contagion. On retrouve aussi des minstrels noirs donnant un numéro de claquettes dans un autre film de Méliès: Les échappés de Charenton, ou l'omnibus des toqués (1901). L'intérêt de cette œuvre n'est pas marginal pour notre propos, car ici la frénésie de leurs mouvements change les minstrels noirs en clowns blancs, qui redeviennent noirs, et ainsi de suite. Ils finissent par éclater en petits morceaux.

\section{HYBRIDATION ET MODERNITÉ}

Ce n'est pas par hasard que la chanteuse épileptique Polaire fut parmi les premières artistes blanches à danser le cake-walk. C'est ainsi qu'elle décrit sa gestuelle et son style de performance dans ses mémoires: «Mon instinct m’a fait faire des gestes follement excités. [...] Je chantais [...] avec mes poings crispés. [...] Je laissais au vent ma toison sauvage [et] chantais avec des mouvements nerveux,

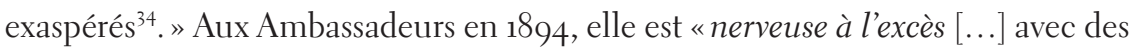
déhanchements fous. Toute cette bruyance instinctive, trépidation incessante. [...] C'est, désormais, le genre Polaire ${ }^{35}$. Polaire est le portrait de l'hystérie; or, pour les 300 ooo spectateurs parisiens qui avaient vu cette même année les danseurs du Dahomey, les termes employés ici ont dû évoquer «la sauvage» avec une

34. Polaire (Émilie-Marie Bouchaud), Polaire par elle-même, Paris, Eugène Figuière, 1933, p. 93 (je souligne).

35. Jean d'Arc, Le courrier français, 22 avril 1894, p. 9 (je souligne). Elle a, de plus, «les seins d'une Almée» et des yeux aux «reflets sauvages» (ibid.). 
insistance égale. D’autant plus que, comme le remarque le metteur en scène du Moulin-Rouge Jacques-Charles :

Le succès de Polaire [lorsqu'elle débutait à la Scala] venait de ce qu'elle était tout à fait différente de toutes les [autres] jolies artistes. Elle n'était pas régulièrement jolie, avec son nez trop gros, sa bouche trop grande, sa peau foncée d'Algérienne, mais ses yeux aux paupières bistrées, aux longs cils étaient admirables. [...] En scène, elle avait une sorte de trépidation nerveuse, un sautillement d'un pied sur l'autre. Le corps nerveux de Polaire, ce corps musclé de petite Arabe faisait sensation. Son type plaisait. Elle fut une devancière ${ }^{36}$.

Sa modernité découle de son hybridité. Pour Nemo et Gondoin, dans «le plus moderne des horizons parisiens», Polaire est «la plus Parisienne d'entre les Parisiennes » tout en étant "plutôt fille d'Afrique à la démarche lente et lascive des femmes de là-bas ${ }^{37}$ ». Colette disait d'elle qu'elle «brillait d'un éclat africain». Cette oscillation entre «la plus Parisienne» et la «fille d'Afrique » résume très précisément l'indécidabilité raciale et culturelle qui a fait de Polaire l'une des plus grandes stars de music-hall.

«Je suis une sauvagesse», explique-t-elle, avant de poser la question : «La sauvagesse est devenue non seulement une Parisienne, mais une des silhouettes les plus représentatives de ce Paris qui réalise tous les paradoxes. Comment cela s'est-il fait ${ }^{38}$ ? N Nous connaissons la réponse: le mélange paradoxal d'une aura primitive, de l'hystérie et du chic parisien est la clé du succès au music-hall. Il y a cependant un prix à payer pour cette hybridité. Les débuts de Polaire datent de 1892, et elle ne tarde pas à devenir la cible des caricaturistes. «Sem, que tous les confrères imitèrent bientôt, me représentait avec un anneau dans le nez et des boucles noires de négresse. [Il a] orné mon corps de rotondités à faire pâlir la Vénus hottentote ${ }^{39}$ ! "Sem a fait pire: dans son album Le vrai et le faux chic, elle est la «suprême horreur, [...] une cannibale crépue [...] le corps étranglé, $[. .$.$] le nez traversé d'un anneau, la bouche sanglante fendue jusqu'aux oreilles { }^{40}$ »

36. Jacques-Charles, Cent ans de music-hall: histoire générale du music-hall, de ses origines à nos jours, en Grande-Bretagne, en France et aux USA, Paris et Genève, Jeheber, 1966, p. 118-119.

37. Nemo et Gondoin, «Soleils couchants », La vie en rose, 26 octobre 1902.

38. «Polaire», Fantasio, 15 novembre 1909, p. 272-273.

39. Polaire, 1933, p. 93.

40. Sem (pseudonyme de Georges Goursat), Le vrai et le faux chic, Paris, Succès, 1914, p. 6-7. «Les femmes vêtues dans cette nouvelle mode ressemblent tout à fait à des femelles sauvages » (p. 6). 
(Fig. 4). Notons que la modernité de la silhouette fashionable est assimilée dans Le vrai et le faux chic à l'image de la sauvage, et ceci depuis $1897^{41}$.

Le cake-walk de Polaire était-il l'expression de sa propre identité, ou a-t-il mimé l'identification de son public? L'intensité et la spécificité de cet échange à la fin du $19^{\mathrm{e}}$ siècle à Paris sont marquées par la dynamique de l'imitation inconsciente. Quand des identifications raciales s'insèrent dans cet échange, cette dynamique se complique davantage.

When a gesture comes to stand for someone other than its apparent originator, what is the meaning? How does it happen?... Maybe marketing of gestures exhibited this complexity of cultural property: that it was saleable but never exclusive property. You could buy it and you could sell it, but you could not own it. Maybe the step took over the stepper ${ }^{42}$.

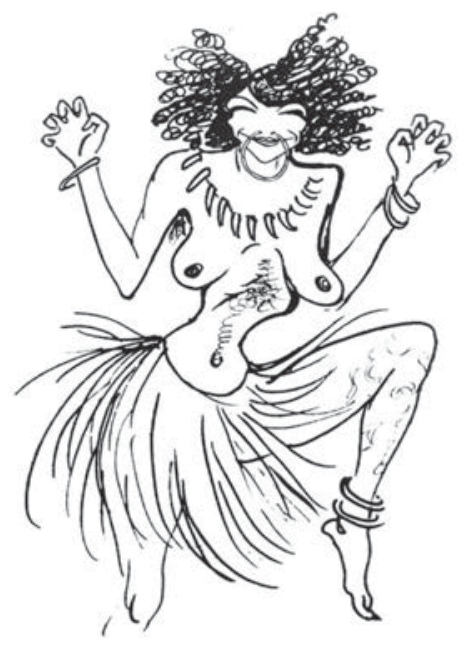

Fig.4: Polaire par Sem, avec la gracieuse permission de la Bibliothèque Forney, Paris.

41. Voir le dessin de Lucien Métivet sur la couverture du Rire du 23 janvier 1897, intitulé «Civilisation! » : un Africain en costume tribal porte «des plumes, des verroteries, des étoffes à fleurs barbares, et des peaux de bêtes », explique la légende. Il est juxtaposé à une Parisienne élégante qui porte... «des plumes, des verroteries, des étoffes à fleurs barbares, et des peaux de bêtes ».

42. W. T. Lhamon, Jr. analyse ici la complexité de la propriété culturelle, Raising Cain: Blackface Performance from Jim Crow to Hip Hop, Cambridge (Mass.), Harvard University Press, 1998, p. 15 (je souligne). Voir son travail brillant sur les minstrels noirs et la gestuelle noire aux USA. 
Outre l'échange entre Polaire et la gestuelle africaine ou noire-américaine, il faut s'interroger sur la nature des échanges entre Polaire et son public. La fascination exercée par l'énergie du corps noir en mouvement est désormais, grâce à la presse, incarnée par la personne de Polaire, par son physique, son lieu de naissance, ses mouvements. La séduction qu'exerce le corps noir sur les Français est ici enrobée d'une peau blanche métissée que le spectateur peut alors désirer ouvertement. De plus, elle façonne les mouvements noirs et épileptiques pour qu'ils correspondent à l'attente de son public. L'expérience des spectateurs est donc celle d'une performance raciale où, à côté de l'identité noire performée, une identité raciale composite, indéterminée - celle de Polaire et d'autres - émerge également. La dynamique entre Noir et Blanc réalisée par le cake-walk est de l'ordre de l'incorporation. Néanmoins, les sentiments résiduels de peur et de répulsion demeurent, et Polaire sera la première à en faire les frais.

\section{CONTAGION}

Entre-temps, les maîtres à danser ont transformé le cake-walk en danse de salon. Maintenant, non seulement les corps des spectateurs au cirque ou au café-concert absorbent inconsciemment les rythmes et les poses du cake-walk, mais les femmes du monde veulent le danser dans leur salon : cet échange entre «haute» et «basse» culture, Blanc et Noir est inquiétant, et bientôt il faudra mettre en garde la bonne société contre le danger de contagion. Sur un ton de badinage, Jacques Redelsperger marie pathologie et évolution dans « Pavane et cake-walk » :

Hélas! Aujourd'hui les méninges des Français sont tout à l'envers, et la cabriole des singes a bouleversé l'univers; Hip! Hip! Hourrah! Qu'on se trémousse, Hardi! Chimpanzés et guenons, chacun y va de sa secousse, [avant de regagner] leurs cocotiers. Le cake-walk épileptique nous fait [...] tourne[r] comme des fous. Laissez suer cette ronde aux abonnés de Charenton ${ }^{43}$.

Un désir de régression s'est-il emparé des Français? La folie du cake-walk s'étend comme une «épidémie» à toutes les couches de la société. Il menace de faire tomber la séparation que les ethnologues avaient soigneusement érigée entre l'homme blanc et le singe, en interposant l'Africain entre les deux.

Un mois après avoir informé ses lecteurs que la version du cake-walk présentée sur les scènes parisiennes n'était qu'une caricature, voici que L'illustration adopte 
un autre ton. À présent, ses pages implorent les mêmes lecteurs d'empêcher cette danse de «s'implanter » et de se propager «dans les salons de bon ton ».

[Avec la valse et le boston ], nous étions encore bien loin de l'acrobatie, des prodiges d'équilibre instable, de la mimique désordonnée et burlesque, caractéristiques de ce cake-walk. [...] Elle est certes fort amusante, cette danse yankee, surtout dans un cirque ou dans un music-hall; cependant [on] ne saurait guère s'en accommoder ailleurs. [...] N'êtes-vous pas là, Mesdames, pour lui clore la porte? Aux excentricités chorégraphiques, aux extravagances épileptiques d'une danse sauvage, vous préférerez des pas raisonnablement réglés et cadencés au rythme d'une musique captivante et... civilisée ${ }^{44}$.

À partir de février 1903, les rythmes et la gestuelle du cake-walk appellent inévitablement la qualification d'épileptique, le plus souvent juxtaposée à celle de «sauvage». W. T. Lhamon, Jr. écrit que le désir de posséder les gestes et les signes de l'autre groupe continue même s'il est maintenant recouvert de dédain ${ }^{45}$. Parmi bien d'autres, un article de l'époque confirme les dires de Lhamon:

[Ce] rythme endiablé d'une musique bizarre, heurtée, incohérente, inharmonique [est pourtant] incontestablement entraînant... [Cependant] dans nos salons, nos jeunes filles ne feraient que perdre en grâce comme en bon ton. Elles ont mieux à danser que ces bamboulas de nègres ${ }^{46}$.

L'ambivalence ici envers le cake-walk est évidente: la moitié de l'article consiste en instructions pour le danser! Notons encore la communication du mouvement aux spectateurs à travers la nature contagieuse (entraînante) du rythme, rappelant les commentaires du Courrier français, de Cocteau, et de tant d'autres.

Le rire avertit ses lecteurs :

Son obsession a gagné toutes les classes de la société, sachez que cette danse nègre fait fureur; les Bals de l'Opéra et de l'Opéra comique ont achevé sa vogue. Aimez-vous le cake-walk? On en a mis partout. Ainsi, ce soir : Bouffes-Parisiennes, Floradora; gh, le cake-walk. Nouveau-Cirque, Joyeux Nègres; 10h, le cake-walk. Odéon, Résurrection, la prison, le cake-walk. Opéra, Siegfried $-5^{\mathrm{e}}$ acte; le cake-walk. Puisqu'il est temps

44. Henri de Rive, «Danses d'autrefois, d'aujourd'hui, de demain», L'illustration, 7 février 1903 (je souligne).

45. «[... continues even if it is now covered over with disdain », dans Lhamon, 1998, p. $76-77$.

46. H. F., 1903, p. 185 . 
encore [assistez au] Grand Meeting de protestation contre le «Mano Negra », encore une danse nègre; il ressemble au cake-walk ${ }^{47}$.

À part chez Wagner et Tolstoï, le cake-walk était réellement omniprésent, et les réunions pour protester se révélaient vaines. C'est le $1^{\text {er }}$ février 1903 et Raoul Ponchon publie le poème «Le cake-walk» en réponse à la nouvelle que les femmes du monde viennent de former la Ligue contre le cake-walk. Dans « cette danse de nos pères, les singes, [il faut] s'agiter et se tordre ». L'apparence du désordre est pourtant trompeuse; les gestes viennent «d'un rite lointain ${ }^{48}$ ». Donc, l'agitation de la danse nous serait léguée de nos ancêtres, les singes! Désormais, la référence à Darwin est de plus en plus de rigueur.

Plusieurs mois plus tard, la couverture de Paris qui chante présente Eugénie Fougère dans «Oh! ce cake-walk» où sont entremêlés danse africaine et noireaméricaine, singes et épilepsie: «On a tout remisé pour les sauts de gorilles. [Avec] des gestes loufoques, votre cerveau bout. Laissons au négro de San Francisco cette ronde épileptique, cette bamboula maboule ${ }^{49}$.» Comme on l'a déjà vu dans "Pavane et cake-walk », le discours de l'évolution se greffe au discours de la psychopathologie. Il en va de même dans «Cak-Walk-Irie» de Mistinguett, où la danse est «la bamboula d'une tribu barbare qui tuait, pillait [...]. C'est fou! Il vous rend maboule ${ }^{50}$.» Pour l'exécuter correctement, «par instants, quelques cris rauques, stridents, sauvagesques sont de rigueur ${ }^{51} »$.

Les références constantes à la gestuelle épileptique constituent, bien sûr, un effort pour comprendre l'étrangeté de la gestuelle noire à travers une étrangeté et un vocabulaire déjà familiers. Regarder danser le cake-walk devait constituer une expérience d'inquiétante étrangeté pour les Français. L'attraction de ces rythmes et mouvements semblait liée au sentiment confus d'une ressemblance à l'Autre par le biais de maladies nerveuses.

W. T. Lhamon, Jr. écrit: «the purpose of popular performance is to include [conflicted messages]; [to] embody conflict and to endow it with hyperbolic excess $^{52} »$. Pour John Szwed:

47. Le rire, 7 février 1903 .

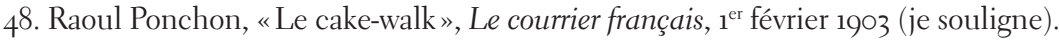

49. Paris qui chante, 18 octobre 1903, paroles Jean Meudrot, musique Édouard Mathé.

50. Paris qui chante, 17 janvier 1904, paroles Serard, musique Chaudoir.

51. H. F., 1903, p. 184.

52. Lhamon, 1996, p. 142. 


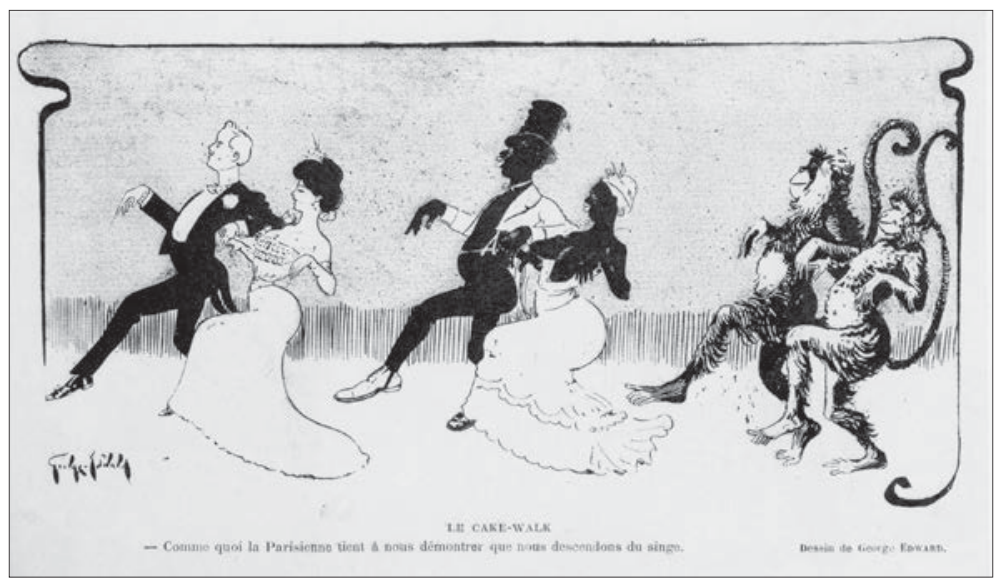

Fig. 5: «Comme quoi la Parisienne tient à nous démontrer que nous descendons du singe », par George Edward, avec la gracieuse permission de la Bibliothèque Forney, Paris.

Attempts are made to lock [the high status group's culture] out of reach [but] the high do learn and borrow from the low... [skirting] the degradation... by a technique that might be called minstrelization; the process by which you can emulate the Other in a socially approved context ${ }^{53}$.

Cependant, malgré la retenue exprimée par l'attitude et l'expression du beau monde à la mode d'après les photographies et lithographies de l'époque, les mouvements et poses du cake-walk ne signifient pas moins la régression. Cette «danse avec Darwin » est effectivement dangereuse : non seulement elle est assimilée à l'épilepsie et à la régression, mais en plus elle brise les barrières de classe et de race. Car c'est bel et bien la Parisienne - symbole d'élégance et de raffinement - qui mène la danse, répandant la contagion et la régression. Cela est insupportable, d'où la création de la Ligue contre le cake-walk (Fig. 5). Aussi raciste que ce dessin nous paraisse - et il l'est certainement -, il faut le replacer dans le contexte de l'anthropologie contemporaine. Les anthropologues du $19^{\mathrm{e}}$ siècle ont permis aux Blancs d'intérioriser une séparation d'avec leurs parents primates en traçant des analogies entre les mouvements, le port et les expressions faciales de ces derniers et ceux des Africains. Ici, pourtant, la haute société parisienne est impliquée dans la généalogie de l'homme ${ }^{54}$.

\section{Szwed, 1975, p. 25 et 27.}

54. Rappelons que la façon dont le corps était maintenu était, au tournant du siècle, un signe de classe très visible. Une pédagogie de contrôle corporel à travers la gymnastique avait pour but un port très droit, pour ne pas dire rigide. 


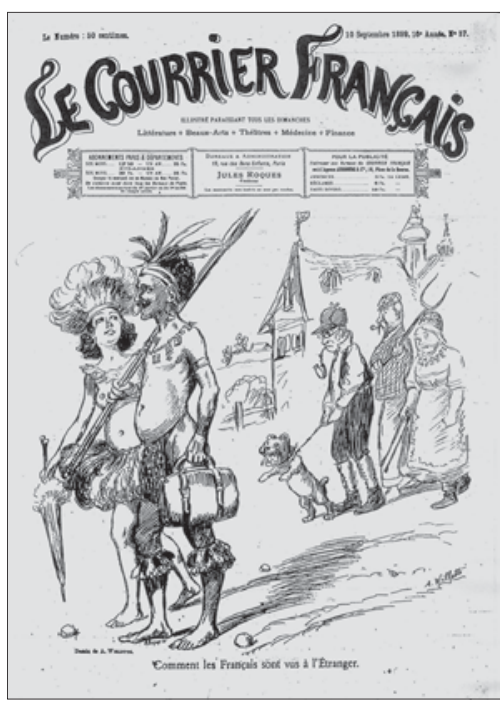

Fig. 6: «Comment les Français sont vus à l'Étranger », par Willette, avec la gracieuse permission de la Bibliothèque Forney, Paris.

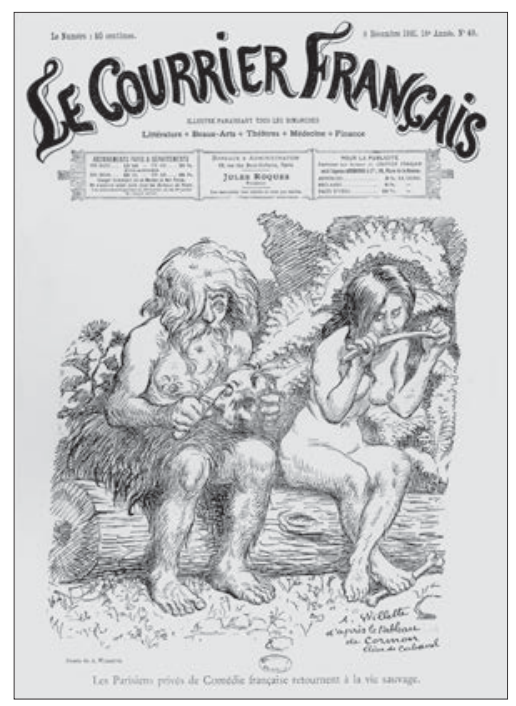

Fig. 7: «Les Parisiens, privés de la Comédie française retournent à la vie sauvage», par Willette, avec la gracieuse permission de la Bibliothèque Forney, Paris.

Naturellement, on craint que cette danse qui brouille les frontières entre Noirs et Blancs n'en vienne, en fin de compte, à détruire ces distinctions. Résultat: tout le monde deviendra sauvage. Or, dans cet interstice entre civilisation et sauvagerie, niche une autre spécificité française. Paris est connu pour l'indécence du cancan, ses revues de danseuses quasiment nues et ses cartes postales pornographiques. En effet, les Français sont perçus par leurs voisins européens comme licencieux, voire comme des obsédés sexuels: ils sont déjà exotiques! Cet étalage des instincts est représenté en 1899 dans un dessin de Willette (Fig. 6). La situation est encore plus dramatique dans ce dessin de 1901: un couple, assis dans une forêt, hirsute et nu, est en train de ronger des os et un crâne humain. Ils sont même devenus cannibales! La raison de cette déchéance se trouve dans la légende: «Les Parisiens privés de la Comédie française retournent à la vie sauvage. » Il suffit de peu de chose: enlevez le vernis de la grande culture, et les Parisiens régresseront à la sauvagerie de l'époque préhistorique (Fig. 7). 


\section{LA DANSE NATIONALE DE FRANCE}

En avril 1903, Le rire publie un dessin de Rodriguez représentant le président Loubet en visite officielle en Afrique du Nord. «Loubet décerne les palmes [au] singe, créateur de notre cake-walk national. Qui donc prétendait que le cake-walk était d'origine américaine?» Le monde illustré du 21 février 1903 constate avec perplexité qu'en dépit de tous les avertissements, le cake-walk a bien triomphé. "C'est une danse apportée du Gabon à la Guadeloupe; les nègres du Gabon l'auraient apprise des singes avant de nous l'enseigner. À toi, Darwin! [...] C'est donc l'art nègre qui triomphe en ce moment... Et voici que nous acceptons le cake-walk comme danse nationale! Pourquoi ${ }^{55}$ ? On trouve la réponse dans le dessin humoristique «L'infériorité de l'Homme ${ }^{56}$ ». Une foule d'animaux - chiens, chats, rats, singes, grenouilles, ours, et un kangourou - sont dessinés en train de danser le cake-walk: tous sont plus agiles que le couple qui le danse dans la dernière image. L’infériorité de l'homme (blanc) renvoie en effet à son degré plus élevé de civilisation, c'est-à-dire à sa difficulté à laisser le corps exprimer les mouvements disloqués, hyperboliques - mais agiles - du cake-walk. Ce qui pouvait se comprendre comme supériorité devient infériorité, un défaut biologique d'énergie ou d'agilité. Les Noirs dansant le cake-walk aux États-Unis sont décrits comme « des coqs en rut. Ils en ont d'ailleurs le tempérament irrésistiblement libidineux. » De plus, le Noir est «un étalon professionnel intrépide auquel la nature prodigue n'a su refuser aucun avantage». En effet, il pourrait apporter «la réponse à l'inquiétant problème [français] de la population [décroissante] qui nous mine ${ }^{57}$ »! Comme on l'a vu dans la publicité pour Kola Marque, la vigueur de l'Africain se superpose à la neurasthénie du mâle français; la chute du nombre des naissances est attribuée à cette carence d'énergie, une énergie qui est, en revanche, bien présente dans les danses africaines et dans le cake-walk électrique, magnétique, «animalistique » et comique. L'ironie que l’on peut relever dans les mots «danse nationale» ne doit pas cacher l'envie. Car cette ressemblance à l'Autre est à la fois crainte et désirée. Il y a une ambivalence considérable entre le mépris (voire la répulsion) et l'envie de la part des Français envers le «primitif»-foyer d'énergie, d'agilité, de liberté corporelle, de vie instinctuelle

55. H. F., 1903, p. 184 .

56. Dessin d'Henriot, Le monde illustré, 7 février 1903, page sans numéro.

57. Harry R. Trémont, «Les nègres du théâtre», Fantasio, ${ }^{\text {er }}$ août 1910, p. 28-29. Trémont constate aussi que le cake-walk aux USA est un «réel concours d'élégance » et «non pas une de ces grotesques contorsions qui ont sévi dernièrement dans nos salons » (p. 27). 
et de puissance sexuelle - tout à l'encontre du label de dégénérescence qu'on a plaqué sur lui. En fait, quoiqu'elle puisse être qualifiée d'«animalistique, primitive et sauvage ", cette énergie vitale et régénérative attise le désir des Français. Cet état de fait devient évident à partir de 1925 avec Joséphine Baker et La Revue Nègre, mais tout semblait déjà tracé deux décennies plus tôt ${ }^{58}$. Ainsi, en 1903, on pouvait déjà lire que « La race noire semble vouloir - ô ironique retour des choses d'ici-bas! - apporter à la race blanche les bienfaits de sa civilisation ${ }^{59}$.»

Danser le cake-walk représente sans doute l'espoir de dépasser la peur de l'Autre en maîtrisant ses mouvements et ses gestes. Or, comme Raoul Ponchon l'a noté dans son poème «Le cake-walk», cette maîtrise manque au Français. Déjà en 1881, dans le Roman d'un spahi, Pierre Loti admirait les complications rythmiques des Griots que «nos organismes [nerveux] européens ne nous permettent pas de parfaitement comprendre ${ }^{60} »$. Il en va de même pour le ragtime: le critique de danse André Levinson écrit en 1927:

Negro ragtime positively dazzles European audiences. This dancing with its automaton-like quality, its marvelous flexibility and its rhythmic fantasy, is as impossible for us to reproduce as it is astounding for us to watch. [...] [This] gift has become more or less atrophied in the cultivated human being. Such is the ransom we pay to civilization ${ }^{61}$.

C'est cela que les corps des chanteuses épileptiques Polaire et Fougère contredisent lorsqu'elles dansent le cake-walk, et c'est précisément ce qui les a rendues si fascinantes, troublantes et également si racialement ambiguës.

Le journaliste qui, le 2 novembre 1902, a prédit une longue et superbe carrière au cake-walk avait raison - plus qu'il ne pouvait imaginer : cette danse est encore bien présente en 1907. La manie n’est pas limitée à la scène. Les frères Lumière ont aussitôt filmé les Elks dans Le cake-walk au Nouveau-Cirque

58. Le livre magistral de Jody Blake étudie l'influence des spectacles populaires du début du $20^{\mathrm{e}}$ siècle sur la peinture et la sculpture modernistes, accordant au cake-walk une place importante au commencement de la série de danses-phénomènes de société culminant avec le tango et le charleston. Elle ne traite ni des correspondances avec le darwinisme, ni de celles avec l'hystéro-épilepsie. Voir son ouvrage, Le Tumulte Noir: Modernist Art and Popular Entertainment in Jazz-Age Paris, 1900-1930, University Park, The Pennsylvania State University Press, 1999.

59. «Le cake-walk des Négrillons», 1903.

6o. Les chants sont accompagnés d'un contretemps «hérissé de difficultés et de bizarreries» ainsi que de «syncopes inattendues». Pierre Loti, Le roman d'un spahi [1881], Paris, Gallimard, coll. «Folio », 1992, p. 135-136.

61. Levinson, 1927, p. 287. 
en 1902; en 1903, en plus du film de Georges Méliès, Pathé produit Le célèbre cake-walk par les Elks, et Le cake-walk des nains, alors qu'Alfred Lubin tourne Le cake-walk.

En 1905 le Nouveau-Cirque joue la carte de l'authenticité avec Les joyeux Nègres avec le vrai cake-walk. Dans son compte-rendu, le Courrier français rappelle les mots d'un certain M. Bergeret: «Les Noirs ont un vif sentiment de la musique. Il se peut qu'il naisse un délicieux art nègre de la danse et du chant. » Cet art nègre sera le jazz. En attendant, depuis novembre 1902, plus de 400 performances des Joyeux Nègres ont continué à enthousiasmer Paris. Mais remarquons comment l'anxiété darwinienne et les critiques moralisantes et esthétiques ont entièrement disparu:

Le public a revu avec plaisir ce spectacle d'un charme si pittoresque et si passionnant: surpris et amusé à nouveau, il a goûté la primitive et prenante poésie de cette chorégraphie, par moments étrange, mais d'une vivacité, une fantaisie, et une gaieté sans pareille [...]. Le public enthousiasmé a applaudi, et frénétiquement crié bravo! et $b i s^{62}$ !

Le cake-walk possède encore la secousse physiologique excitante de la surprise, mais son «étrangeté » est maintenant acceptée comme une forme de poésie primitive.

La passion des Français pour les rythmes et gestes de la danse noire n'a pas pris fin lorsque la vogue du cake-walk s'est épuisée. Bien des années plus tard, Yvette Guilbert déplore ce fait: «Le nègre est devenu "l'Inspirateur français"! Sa musique, ses chants, ses danses, [...] et jusqu’à sa plastique déhanchée, déclenchée, tout de lui devient: Modèle pour Français! » La contagion des mouvements pathologiques est alors explicitement attribuée au rythme: «La musique nègre a désaxé [le Français], lui, sa femme, ses enfants se dandinent, se déhanchent, aux sons d'un disque nègre, et l'âme noire entre en eux, comme un point épidémique de variole, et leur inocule son virus africain ${ }^{63}$ ! Exprimant les mêmes inquiétudes sur un ton beaucoup moins violent, André Levinson écrit:

Negro dancing may suggest $[\ldots]$ quite separate problems. [...] The moralist may be more concerned with the effect of this black virus upon European civilization $[\ldots]$ while, as it is found in our present-day dance halls, it may appear as a symptom of an epidemic contagion of society which should concern the pathologist $t^{64}$.

62. «Nouveau-Cirque», Le courrier français, 16 mars 1905, p. 4.

63. Yvette Guilbert, Une heure de musique avec Yvette Guilbert, Paris, Éditions Cosmopolites, 1930, p. 9 et 11.

64. Levinson, 1927, p. 282. 
Le texte de Levinson condense bien l'influence de l'anthropologie et de la médecine dont la coalescence était si frappante à la Belle Époque qu’elle trouve écho dans les écrits savants aussi bien que dans les chansons populaires.

L'emprise du rythme échappe souvent au contrôle du danseur. Une fois libérée, l'énergie prend son élan et vit de sa propre vie; le corps automatique s'affranchit de la conscience dans cette extraordinaire libération de libido, d'instinct, de mouvement. Le supplément de la race tient ces caractéristiques le plus parfaitement de la danse noire - ou, il serait plus juste de dire : à la danse noire comme elle était perçue en France. Le désir d'imiter ou d'incorporer ces rythmes est en effet puissant, et les stars parisiennes blanches qui se sont approprié le cake-walk ont construit une image hybride de noir et de blanc qui deviendra un élément important des avant-gardes du $20^{\mathrm{e}}$ siècle $^{65}$.

\section{CHOCOLAT REDUX: AN AMERICAN IN PARIS}

Grâce au cinéma, la vie et le mouvement animent la lithographie de 1896 de Toulouse-Lautrec, "Chocolat dansant dans un bar». Dans l'ultime séquence de ballet du film de Vincente Minnelli, An American in Paris (1951), le personnage interprété par Gene Kelly (Jerry Mulligan) incorpore littéralement - et donc devient - Chocolat en superposant son corps à celui, noir, de la lithographie le représentant. Kelly-Chocolat danse entre les reproductions en carton des images que Toulouse-Lautrec nous a laissées d'Yvette Guilbert, de la Goulue,

65. À titre d'exemples: Ubu colonial d'Alfred Jarry, les poèmes nègres d'Hugo Ball, les danseuses de Rudolf Laban habillées en négresse, le Maure - un danseur en blackface - dans la séquence des «Scènes burlesques» dans Перушка (Petrouchka, 1910-1911) de Stravinsky, et le film Le nègre blanc (production Albatros, restauré en 2009-2010 d'après 2 copies Pathé-Baby, 1925) de Nicolas Rimsky et Henry Wulschleger. Rimsky, comique accompli, joue un contrebassiste de music-hall parisien qui est invité à jouer au château d'un riche Américain. Arrivé au château après des péripéties, il est hypnotisé par une sculpture de chef africain aux yeux lancinants, et rêve qu'il est devenu un sauvage blanc. Arrive l'Académie des Sciences surnaturelles, avec un savant qui prend ses mesures anthropométriques qui déterminent qu'il a le crâne atrophié. L’Américain organise une fête pour présenter «son Nègre blanc» à des amis. «Il se comporte, naturellement, comme un sauvage », nous dit l'intertitre. Affublé de plumages, il exécute une «danse de son pays », «en sautant et en jetant ses pieds dans tous les sens pendant qu'il brandit sa hache... surtout contre le chef d'orchestre. Cette "Danse de la hache" effraye et en même temps fait rire les convives. Un gros plan nous le montre apeuré par leur regard : il les menace en criant, “Arrière les vrais Blancs”.» Les films Pathé-Baby étaient destinés à des projections en famille. Il est à noter que ce film est sorti quinze jours après la première de La revue Nègre. 
de Valentin le Désossé, et des spectateurs comme Oscar Wilde, appartenant tous au fameux décor du Moulin Rouge. Comme on le sait à présent, le music-hall parisien, dont le Moulin Rouge est le symbole même, exhibe le corps totalement libéré, aussi bien qu'une coalescence parfaite de gestes noirs et blancs. C'est pourquoi Chocolat doit être au centre de l'extraordinaire clou du spectacle placé au Moulin Rouge. Gene Kelly, le danseur blanc, doit incorporer les gestes du danseur noir afin de les fondre à ceux du cancan typiquement parisien. Dans cette imitation, les fesses de Kelly sont excessivement protubérantes et ses mains miment celles d'un minstrel noir. Après une série de pas de danse acrobatiques, entouré d'un cercle de danseuses de cancan, il devient le partenaire de Jane Avril ${ }^{66}$ dans un cancan érotique. Les dernières images du ballet de An American in Paris montrent le cancan évoluer, se métamorphoser en cake-walk, lui aussi typiquement parisien grâce aux chanteuses épileptiques qui l'ont dansé. Chocolat et Jane Avril gambadent hors de la scène dans un high-stepping cake-walk, quittant le music-hall pour la Place de l'Opéra avec La danse de Carpeaux explicitement mise en valeur. Ce désir d'appropriation du mouvement et de la gestuelle noirs par des artistes blancs a lieu à Paris, pas seulement comme une toile de fond, mais comme le signe même de cette appropriation. Désormais fortement identifiée comme danse parisienne, le cake-walk se greffe, voire se substitue au cancan dans une hybridation exemplaire.

66. Entre quatorze et dix-sept ans, Jane Avril était internée comme hystérique à la Salpêtrière. Son style de danse excentrique entrera en parfaite harmonie avec les chanteuses épileptiques à l'affiche au Moulin-Rouge. 